\title{
Approaches to the Formation of the Basis of Assembly and Management of Robots in the System of Training Bachelors
}

\author{
Tufik Kaziaxmedov ${ }^{1, a *}$, Georgij Yalamov ${ }^{2, b}$ \\ 1 Nizhnevartovsk State University, 628605, Nizhnevartovsk, 56 Lenin str., Russia \\ 2 Institute of Education Management of the Russian Academy of Education, 105062, 16 \\ Zhukovskogo str., Moscow, Russia
}

aktofik@yandex.ru, ${ }^{b}$ geo@portalsga.ru

${ }^{*}$ Corresponding author

Keywords: robotics, educational robotics, microcomputers, microcontrollers, sensors for educational robots, industrial sensors, IT industry, IT specialist, Bachelors

\begin{abstract}
The article describes a number of approaches to the formation of competencies in the field of assembly and programming of robotics, the intellectualization of robotic complexes, the study of these issues in the preparation of IT specialists in two-level training (bachelor-master). Examples of the implementation of educational robotic projects are given, the rationale for the proposed program of the course "Fundamentals of Robotics" in the preparation of Bachelors in Computer Science and Computer Engineering is carried out.
\end{abstract}

\section{Introduction}

The development of the IT industry in Russia is one of the main directions for the development of an innovative economy, which requires the introduction of modern information educational technologies that have been tested in domestic practice and abroad. Related tends include the intellectualization of information systems in all fields of activity of modern society, the implementation of practice-oriented teaching methods, competence and contextual approaches to the education of students.

In papers $[1,2]$ we analyzed the Federal State Educational Standards of Higher Education, the working functions recommended by the professional community of the Russian Federation, functionality of posts at IT enterprises of the Russian Federation, etc. and came to the conclusion that many experts ignore the study of robotics and the use of robotics in the automation of production processes in the training process of Bachelors in the fields of Computer Science and Computing, Information Systems and Technologies.

Today, when it comes to programming, we understand that it also includes programming of mobile devices, microcomputers, and microcontrollers, which act as the "brains" of robots, computer-controlled machine tools.

Robotics and programmable technical devices should contribute to the development of a new innovative industry in our country. Therefore, learning the basics of assembling the simplest robots and studying their use for automating various production processes, aimed at the formation of relevant professional competencies, seems to us to be mandatory for modern IT specialists.

\section{Methodology}

As a course of choice, we recommend the discipline "Basics of Modern Robotics" for Bachelors in the areas "09.03.01 - Informatics and Computing" and "09.03.02 - Information Systems and Technologies," and for future informatics teachers studying the discipline "Educational Robotics."

The appearance of these courses is related to the fact that we participated in the implementation of such regional educational projects as "Smart Home", "Intellectual Field", "Robot Storozh", "Walking Robots", and "Aircraft" in collaboration with students. It was the ability to purchase minicomputers, microcontrollers, various sensors, and parts for robots on the market that enabled the low-cost implementation of the above projects.

The need to train teachers of Computer Science in the field of Educational Robotics is due to the fact 
that in many schools of the Ural Federal District there are so-called technology parks, which are based on robotics.

\section{Research}

To substantiate the proposed program of the discipline "Fundamentals of Robotics," we consider some details of the projects we have implemented. We give the general structure of the project "A Budget Smart Home," which is practically developed and tested in action.

The project consists of hardware and software. The hardware is represented by modules that perform the functions of ready-made blocks for creating the "Smart Home" system. The software is designed to configure the modules and system behavior, control the required parameters, and provide control actions. In this case, all actions can be performed remotely via the Internet. The overall structure of the system is shown in Figure 1.

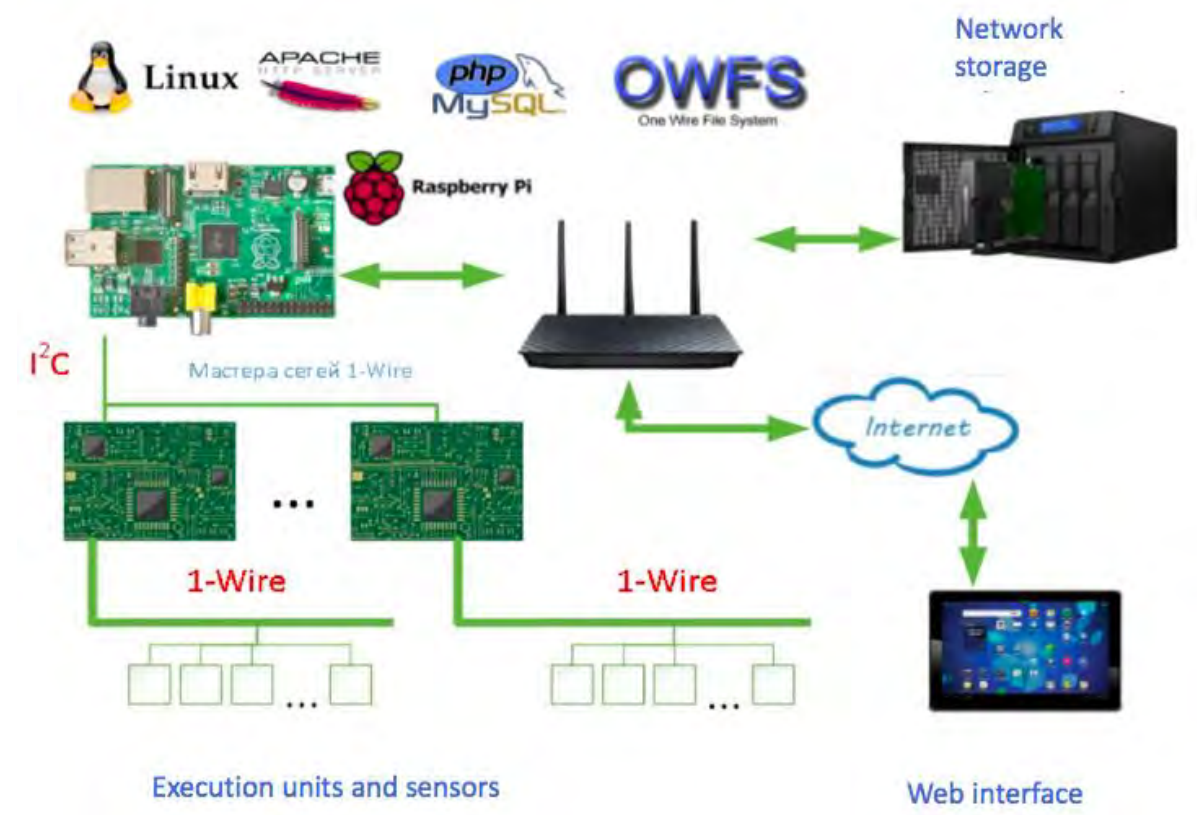

Fig. 1. The general structure of the system "Smart Home."

The Raspberry Pi microcomputer acts as a web server, database server, and single wire network server. If necessary, one can include several microcomputers in the system and assign each individual functions. Through the I2C interface, network masters of the 1-Wire networks are connected to the microcomputer ports. The I2C bus supports the "hot plugging" of about a hundred devices per bus. Next, the end devices are connected to the network masters via a single-wire bus. The operating system for the Raspberry Pi and data are stored on an external plug-in memory card. For more secure and efficient data storage, in the future we plan to include a network storage in the system, which would be located in the local network of the house. It will store and process database files, backups, operating system images for the microcomputer, and other important and necessary information for the system's functioning.

The control program is a web application and is written in PHP. Application scripts are executed on the web server and they are accessed by the database server and end devices via the 1-Wire network server.

The project of implementation of the "Storozh" robot was very interesting. In the course of the project, it became necessary to study various microcomputers used in the security field. We give the characteristics of some of them. APC 8750 microcomputer is demonstrated in Fig. 2. 


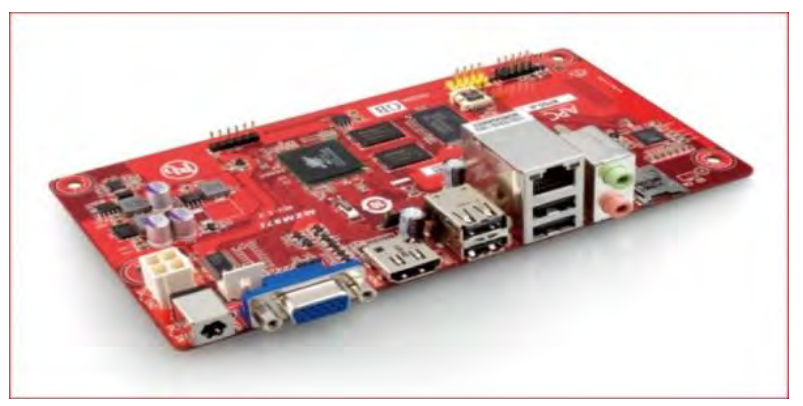

Fig. 2. APC 8750 motherboard.

- VIA WonderMedia processor, $800 \mathrm{MHz}$ with an additional 2D / 3D graphics processing unit up to $720 \mathrm{p}$.

- 512 GB RAM.

- 10/100 Ethernet network controller.

- USB 2.0 ports.

- Slot for microSD.

- HDML and D-sub interfaces.

The microcomputer Raspberry Pi model B 512 Mb (Figure 3).

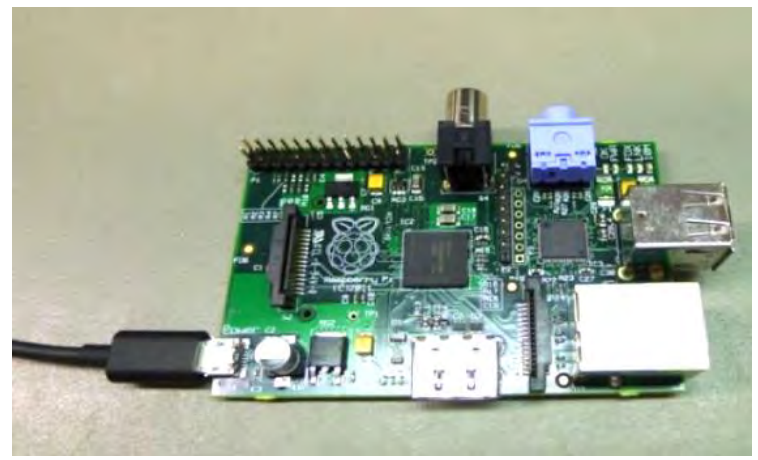

Fig. 3. Motherboard Raspberry Pi model B $512 \mathrm{Mb}$

- ARM1176JZF-S $700 \mathrm{MHz}$ processor with VideoCore IV video accelerator supporting Full HD resolution.

- RAM $512 \mathrm{Mb}$.

- UBS 2.0.

- 10/100 Ethernet network controller.

- SD slot.

- Interfaces: UART, I2C, SPI, ARM JTAG, DSI interface, MIPI CSI-2, GPIO.

IntelGalileo microcomputer (Figure 4). 


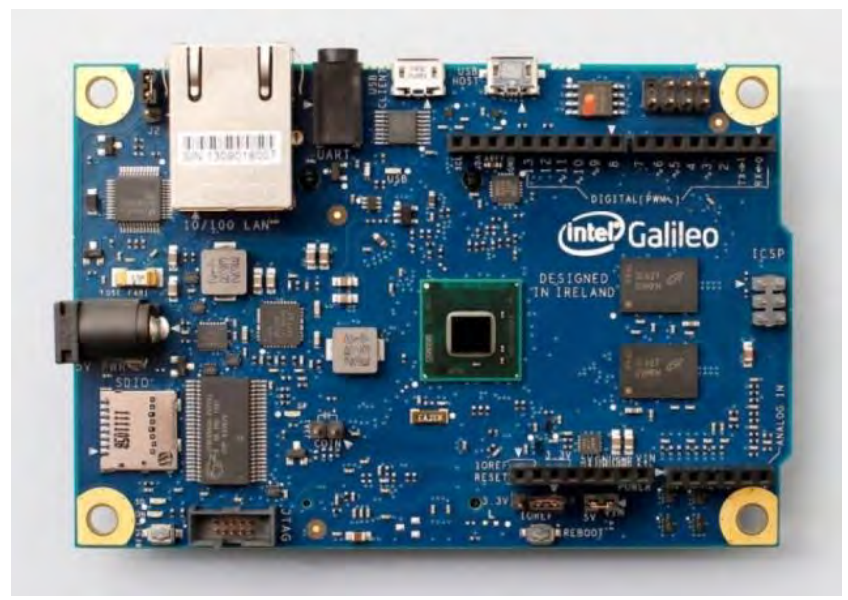

Fig. 4. IntelGalileo motherboard.

- IntelQuark X1000 with a frequency of $400 \mathrm{MHz}$.

- RAM $256 \mathrm{Mb}$.

- Slot USB 2.0 client, slot USB 2.0 server.

- 10/100 Ethernet network controller.

- MicroSD slot.

- Interfaces: RS-232, UART, JTAG, NOR-flash, mini-PCI Express.

Microcomputer A13-OLINUXINO (Figure 5).

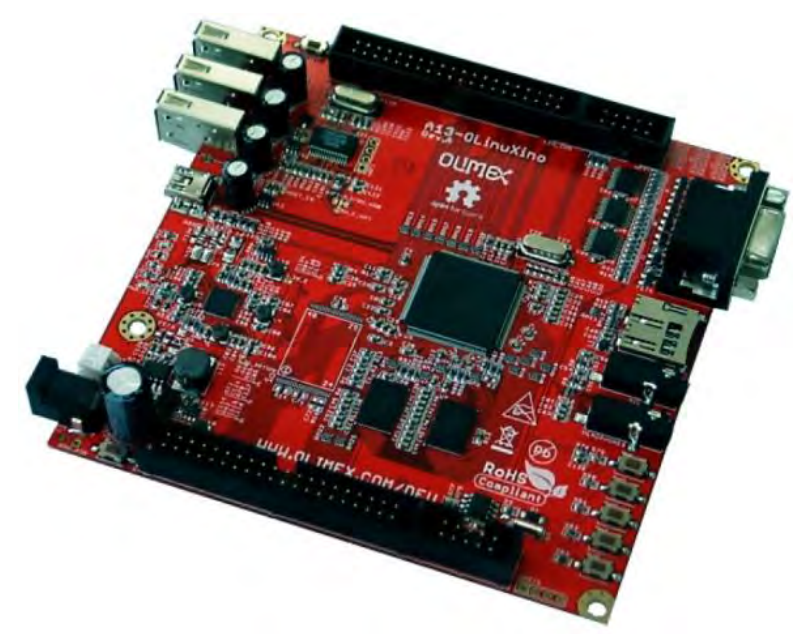

Fig. 5. Motherboard A13-OLINUXINO.

- Allwinner A13 ARM processor with $1 \mathrm{GHz}$, Mali 400 graphics system.

- RAM 512Mb.

- UBS 2.0

- 10/100 Ethernet network controller.

- SD slot.

- Interfaces: UEXT, NAND flash, LCD, HDMI, UART, I2C. 
The Cubieboard2 A20 microcomputer (Figure 6).

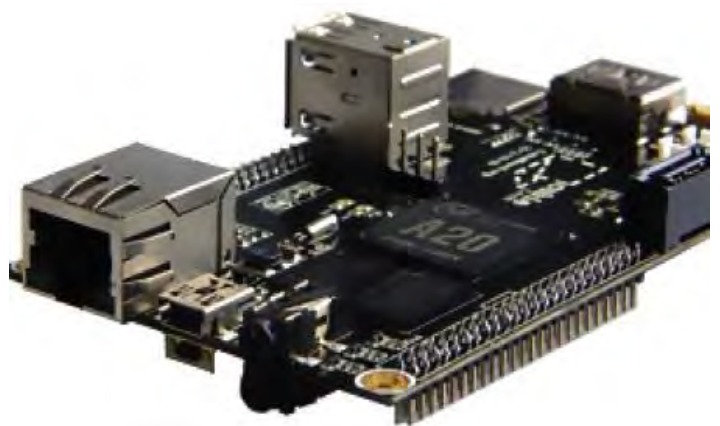

Fig. 6. Cubieboard2 A20 motherboard.

- Dual core 1.6MHz DualcoreARMcortex-A7 processor with NEON, VFPv4 support.

- RAM 512Mb.

- UBS 2.0.

- $10 / 100$ Ethernet network controller.

- MicroSD slot.

- Interfaces: 2C, SPI, RGB / LVDS, CSI / TS, FM-IN, ADC, CVBS, VGA, SPDIF-OUT, R-TP.

To create the "Storozh" robot, we used the Raspberry Pi single-board microcomputer.

Benefits of choosing a Raspberry Pi:

1. Affordable or reasonable price.

2. The ability to connect it to the Internet.

3. Availability of GPIO interface.

4. Wide use for different tasks.

The Raspberry Pi microcomputer in the Storozh robot performs the functions of a control panel.

From the motion sensors, we selected the security optical-electronic "Photon-14 (IO 409-18)" (Figure 7).

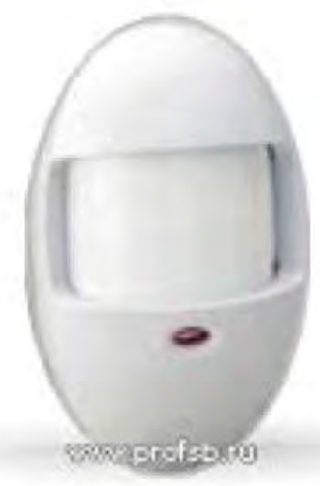

Fig. 7. Motion sensor "Photon-14 (IO 409-18)".

The advantages of our choice of this motion sensor are as follows:

1. Its easy accessibility: such a detector can be purchased at any store of security and fire alarms. 


\section{Low cost.}

3. Easy operation and use.

4. The detection zone $8 \mathrm{mh} 10 \mathrm{~m}$.

5. Supply voltage 9-16 V, current $16 \mathrm{~mA}$.

6. The duration of the alert not less than $2 \mathrm{~s}$.

7. Detection zones: 16 distant zones, 3 medium, 4 near.

8. Sensitivity is selected by the "FEELER" switch.

9. Operating temperature range from -10 to $+50^{\circ} \mathrm{C}$.

10. Relative humidity $95 \%$ at $25^{\circ} \mathrm{C}$ without moisture condensation.

11. Dimensions $67 \times 104 \times 38 \mathrm{~mm}$.

When connecting production-type sensors to the Raspberry Pi microcomputer, it is necessary to take into account that the sensors themselves (detectors) require a minimum voltage of $12 \mathrm{~V}$, and a maximum of $5 \mathrm{~V}$ can be used to power the Raspberry Pi.

For the notification of a fire, a smoke detector "ИП 212-45" was chosen (Figure 8).

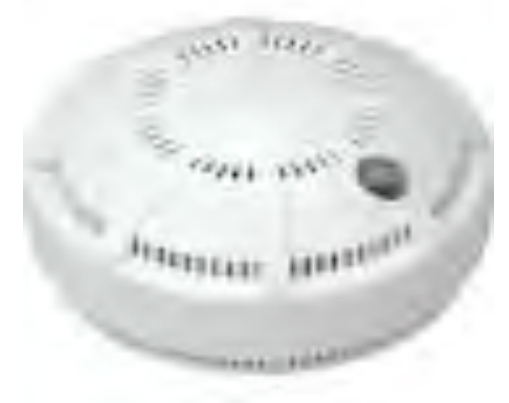

Fig. 8. Smoke detector “ИП 212 -45."

This detector is characterized by easy operation. The sensitivity of the detector is from 0.05 to $0.2 \mathrm{~dB} /$ $\mathrm{m}$, the inertia of triggering of the detector is not more than $9 \mathrm{~s}$, the supply voltage is from 9 to $30 \mathrm{~V}$ and possible polarity reversal of the supply voltage with a duration of $100 \mathrm{~ms}$ and a frequency of not more than $0.7 \mathrm{~s}$. Power consumption current at a voltage of $20 \mathrm{~V}$ not more than $45 \mu \mathrm{A}$.

To connect sensors to the Raspberry Pi microcomputer, we used a GPIO port - this is a universal software-controlled I / O port consisting of several connectors.

In the Raspberry Pi microcomputer, the GPIO port does not have a specific purpose, and the user can use it at his discretion.

- The GPIO port of the Raspberry PimodelB consists of:

- Outputs with a constant voltage of $5 \mathrm{~V}$;

- 2 outputs with a constant voltage of $3.3 \mathrm{~V}$;

- Common ground leads;

- 17 digital software-controlled connectors.

Figure 9 shows the pinout of the GPIO port and the designation of connectors and pins. 

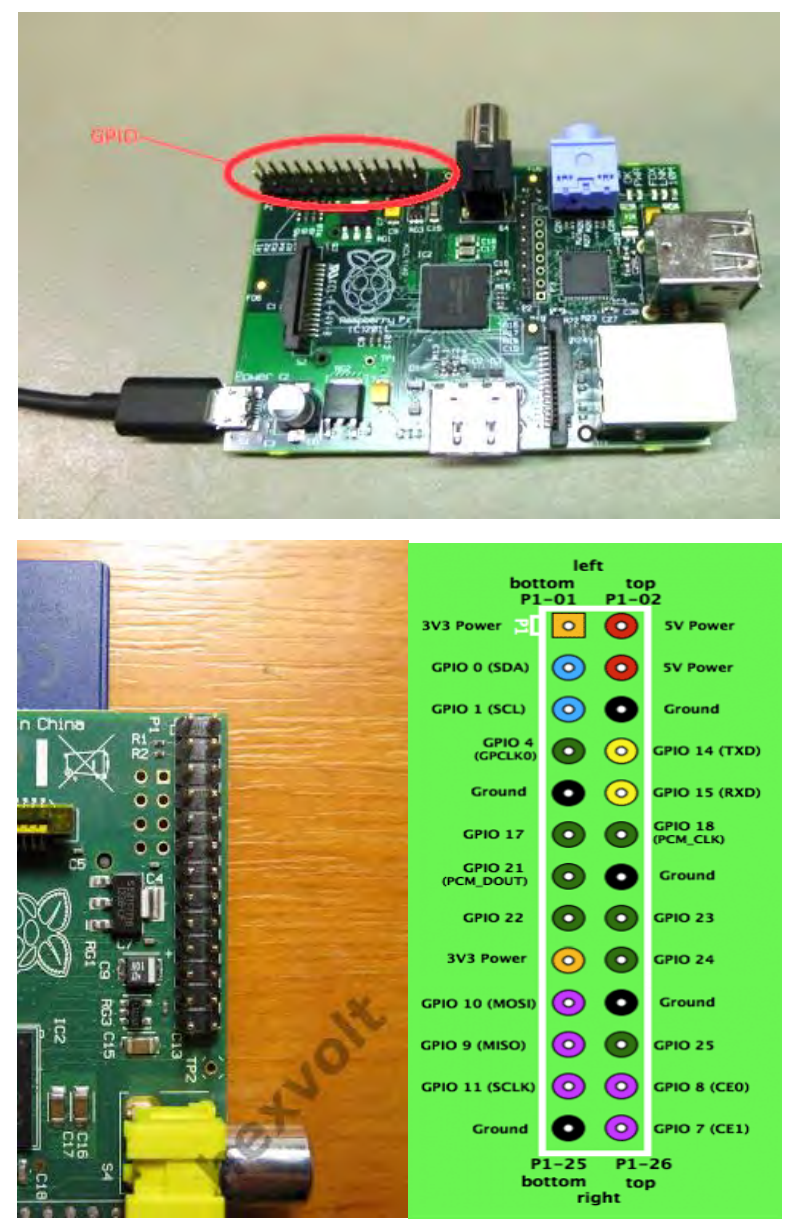

Fig. 9. The Pinout GPIOport.

- 3V3 Power: a port with a 3.3V output signal.

- 5V5 Power: a port with $5 \mathrm{~V}$ output signal.

- Ground is a common ground connection sweat.

- GPIO - 3.3V Universal I / O Port.

Each GPIO port is universally programmable and can be used both as an input and an output. Some GPIO ports also have alternative functions:

- SDA, SCL - I2C interface pins.

- TXD, RXD - UART pins.

- MOSI, MISO, SCLK, CE0, CE1 - SPI interface pins.

- GPCLK0 - (GeneralPurposeClock) output for forming a variable clock frequency for external devices.

- PCM_CLK, PCM_DOUT - I2S audio interface outputs.

A $12 \mathrm{~V}$ power supply is used to power the sensor, and the sensor itself is connected to the 7 th and 8 th connectors.

To transfer frames from the camera over the network to the web browser, we installed and configured the free program "Motion".

The "Motion" is a video surveillance program for Linux. Works with webcams via video4linux and with network IP cameras. Monitors the signal received from one or more cameras, and, when changes are 
detected in the picture, records photos in JPEG or MPEG video streaming formats. Capture frames at regular intervals. Event information can be sent to a specified email address, upon the occurrence of an event (motion detection), custom commands or a script can also be executed.

Let's take a chart of the assembly of the robot "Storozh" (Figure 10).

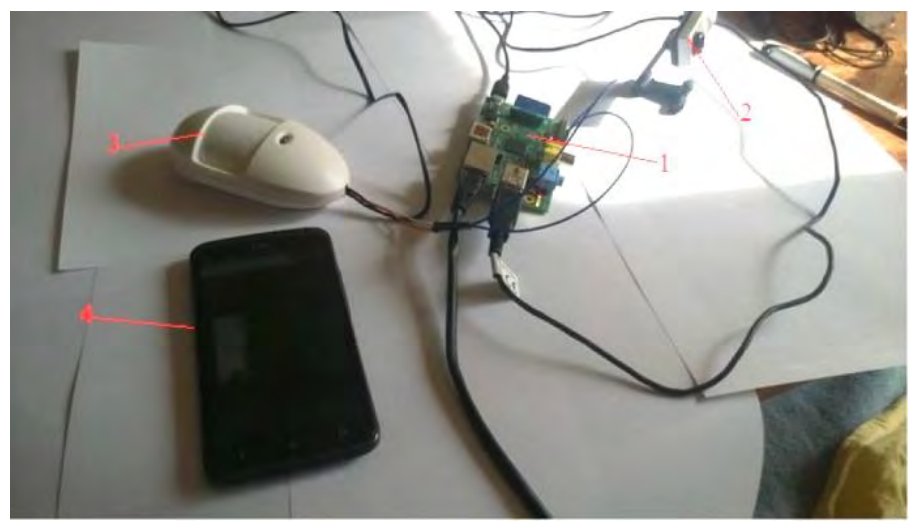

Fig. 10. The assembly scheme of the robot "Storozh": 1. - Reception and control device (Raspberry Pi). 2. - USB webcam. 3. - Motion sensor. 4. - Central monitoring console (telephone).

A webcam is connected to the Raspberry Pi via a USB port. The image is broadcast from it constantly. Video is broadcast over the network through the program "Motion." On the phone through a web browser, one can get a picture. Also, the Raspberry Pi is connected to the security detector "Photon 14." The sensor is connected as follows (Figure 11):

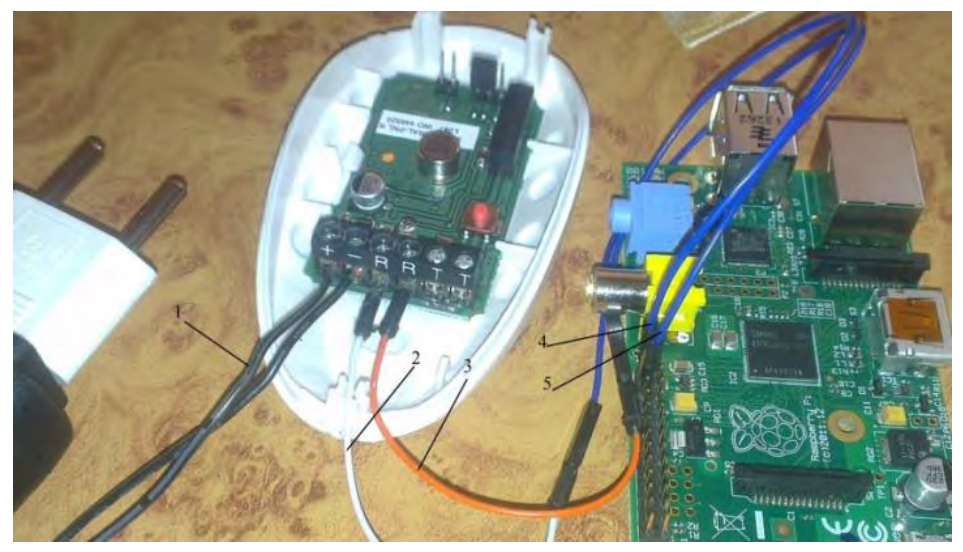

Fig.11. Connection diagram of the Foton-14 motion sensor to the Raspberry Pi microcomputer. 1. - Power. 2. - The output of the loop. 3. - The input loop. 4. - 7 GPIO port. 5. - 8 GPIO port.

For the full-fledged operation of the "Photo-14" security detector, an electric current with a voltage of 9 to $30 \mathrm{~V}$ and a current of $1 \mathrm{~mA}$ are required. This function provides a connected $12 \mathrm{~V}$ power supply with a current of $1 \mathrm{~A}$. This is more than enough to power the sensor. From the GPIO port 7, an electric current is supplied corresponding to a logical unit of $3.3 \mathrm{~V}$ and the GPIO 8 port receives this current. The principle of operation is as follows: when the detector detects the movement of the infrared background in its vision zone, the electric current supplied from the power supply passes through the output relay and, passing a current through the coil, creates a magnetic induction that saturates the armature; therefore, the loop circuit opens and closes on the GPIO 8 port. With the help of the program, the change of the signal is fixed, it starts to vary from 0 to 1 . The robot developed by us has an autonomous power supply system with solar batteries. It has been tested in practice in the protection of summer cottages and has a good commercial perspective.

The given examples show that knowledge of modern sensors and their protocols, microcomputers, and their software is additionally required to study robotics. In this regard, the course "Basics of Robotics" offered by us includes the following sections.:

1. "Modern Microcomputers and Microcontrollers, Their Software."

This section covers the study of the characteristics of the microcomputers listed above, the devices 
included in these boards, their software, including installation and configuration, including the installation and configuration of Arduino in operating system of Windows.

2. "Modern Industrial Sensors. Sensors for Robotics."

3. "Common Robotic Kits (Constructors).

More information about the course program can be found in [3].

This section examines 1-2 constructors and builds robots. Programs are written for robots (providing intelligence). Designers are selected from the following depending on their availability:

- Robots that can be built using EV3 (LEGO).

- Designer FischerTechnik ("Fischertechnik GmbH," Germany).

- Designer Arduino.

- Android robots designer Bioloid.

4. "Fundamentals of Mechatronics."

\section{Conclusion}

The methodological feasibility of the approaches mentioned above is due to their focus on the implementation of a training type of activity. A student not only learns knowledge, but independently acquires the necessary competencies, delves deeper into the essence of the question, he has an interest in the subject. He actively uses educational and technical literature, works out algorithms of professional actions. The principles and methods of developmental education are being implemented. A student gets an opportunity to apply their own methods and techniques.

The use of satellite technologies, mobile devices, robotics in the development of information systems and software systems yields positive results in the field of the quality of training future IT specialists. As our experience shows, to obtain the desired result, it is necessary that the teachers of Physics, Mathematics, Chemistry, and Biology also take part in this work. We all really need to work to train specialists in the modern information technology community for such an important area as the IT industry.

\section{References}

[1] Kaziakhmedov, T. B. (2014). Advance training in the field of IT-industry in a developing economy and long-term reforms in higher education. Pedagogical Computer Science, 4, pp. 62-72.

[2] Vagramenko, Y. A., Kaziakhmedov, T. B., Yalamov, G. Y. (2017). Pedagogical and technological aspects of the intellectualization of the information environment, which includes objects of educational robotics. Informatization of Education and Science, 3(35), pp. 75-82.

[3] Kaziakhmedov, T. B. (2017). Methodical approaches to teaching robotics bachelors in the direction of training 09.03.01 computer science and computing. Journal of European Social Sciences, 9, pp.146159. 